\title{
Effects of LO Phase and Amplitude Imbalances and Phase Noise on $M$-QAM Transceiver Performance
}

\author{
ZhenQi Chen, Student Member, IEEE, and Fa Foster Dai, Fellow, IEEE
}

\begin{abstract}
This paper presents a rigorous analytical model for analyzing the effects of local oscillator output imperfections such as phase/amplitude imbalances and phase noise on $M$-ary quadrature amplitude modulation ( $M$-QAM) transceiver performance. A closed-form expression of the error vector magnitude (EVM) and an analytic expression of the symbol error rate (SER) are derived considering a single-carrier linear transceiver link with additive white Gaussian noise channel. The proposed analytical model achieves a good agreement with the simulation results based on the Monte Carlo method. The proposed QAM imperfection analysis model provides an efficient means for system and circuit designers to analyze the wireless transceiver performance and specify the transceiver block specifications.
\end{abstract}

Index Terms-Amplitude imbalance, error vector magnitude (EVM), local oscillation (LO) generation, phase imbalance, phase noise, quadrature amplitude modulation (QAM), symbol error rate (SER), transceiver RFIC.

\section{INTRODUCTION}

W ITH the ever-increasing demand for a high data rate in emerging communication systems such as thirdgeneration $(3 \mathrm{G})$ wireless systems, wireless local area network (WLAN) systems, and digital video broadcasting (DVB), high bandwidth efficiency is highly demanded. An $M$-ary quadrature amplitude modulation ( $M$-QAM) with a large $M$ value such as 32,64, or 128 has received increasing interest for many wireless communications. However, as the price paid for the high bandwidth efficiency, the $M$-QAM systems with a large $M$ value place more stringent requirements than the slow-datarate 4-ary or 8-ary modulations on many wireless transceiver specifications such as the SNR, linearity, local oscillation (LO) phase and amplitude accuracy, LO synchronizations, and LO phase noise.

On top of the complicated modulation schemes, the emerging high-data-rate wireless systems are required to operate at higher and higher frequency bands, e.g., $2 \mathrm{GHz}$ for $3 \mathrm{G}$ systems, 2.4/5.2 GHz for WLAN networks, and $3 \mathrm{GHz} \sim 10.6 \mathrm{GHz}$ for ultra-wideband applications. Therefore, high-data-rate wireless transceiver designs are facing increasing challenges to provide clean and balanced LO quadrature signals. For a typical voltage-controlled oscillator (VCO), the phase noise increases with the square of the center frequency [1], [2]. Therefore, at a

Manuscript received January 20, 2009; revised January 29, 2010. First published February 8, 2010; current version published April 14, 2010.

The authors are with Auburn University, Auburn, AL 36849 USA (e-mail: chenzhe@auburn.edu; daifa01@ auburn.edu).

Color versions of one or more of the figures in this paper are available online at http://ieeexplore.ieee.org.

Digital Object Identifier 10.1109/TIE.2010.2042417 high frequency band, the phase noise effect is rather significant for the $M$-QAM systems with a large $M$ value. Furthermore, the quadrature synthesizer will introduce non-negligible phase and amplitude imbalances for the LO frequency in the gigahertz range. Those imbalances will further degrade the QAM system performance [12]. Because the free-space propagation loss is proportional to the square of the frequency [3], higher transmission power is needed for the increased range and receiver sensibility. The coexistence of multiple wireless standards also generates a more complicated wireless environment. The resultant degrades the wireless spectrum with large jamming signals, and interferences have placed an increased linearity requirement for the receiver front-end designs.

The link budget and system parameters have to be carefully considered and budgeted into the transceiver block specifications. The $M$-QAM places stringent wireless system requirements that increasingly challenge the transceiver RFIC designs. It is thus desirable for both system- and circuit-level designs to have a rigorous analytical model for the imperfections of the transceiver such as phase and amplitude imbalances, phase noise, nonideal synchronizations, and nonlinearity. Those imperfections directly affect the QAM system performance. There have been investigations related to this topic. Several theoretical models of the VCO phase noise have been derived in [4]-[7]. Furthermore, from a practical point of view, the contributions from different noise sources in a phase-locked loop (PLL) frequency synthesizer design for WLAN applications have been modeled in [8], which can be used to simulate the overall PLL synthesizer phase noise performance. A quadrature PLL will also introduce phase errors between two quadrature channels in QAM. [14] An early-developed model of the phase and amplitude imbalances and dc offset in the quadrature modulator and demodulator has been reported in [9]. The $M$-QAMorthogonal frequency-division multiplexing system performance in the presence of a nonlinear amplifier and phase noise is analyzed in [10]. The error vector magnitude (EVM) is normally used as a major system specification to quantify the QAM accuracy. The work presented in [11] derives the effects of imbalances and phase noise of the LO signals on the EVM. The work given in [12] discussed an approach to apply EVM to RF system design. However, it is the symbol error rate (SER) that is directly related to the system performance of a transceiver data link, and the relationship between the SER and the EVM is not straightforward. So far, the effects of the imbalances and imperfections on the SER have not been discussed and analytically modeled in previous publications.

In this paper, a system model of a transceiver data link for an $M$-QAM system is built, which includes the phase 


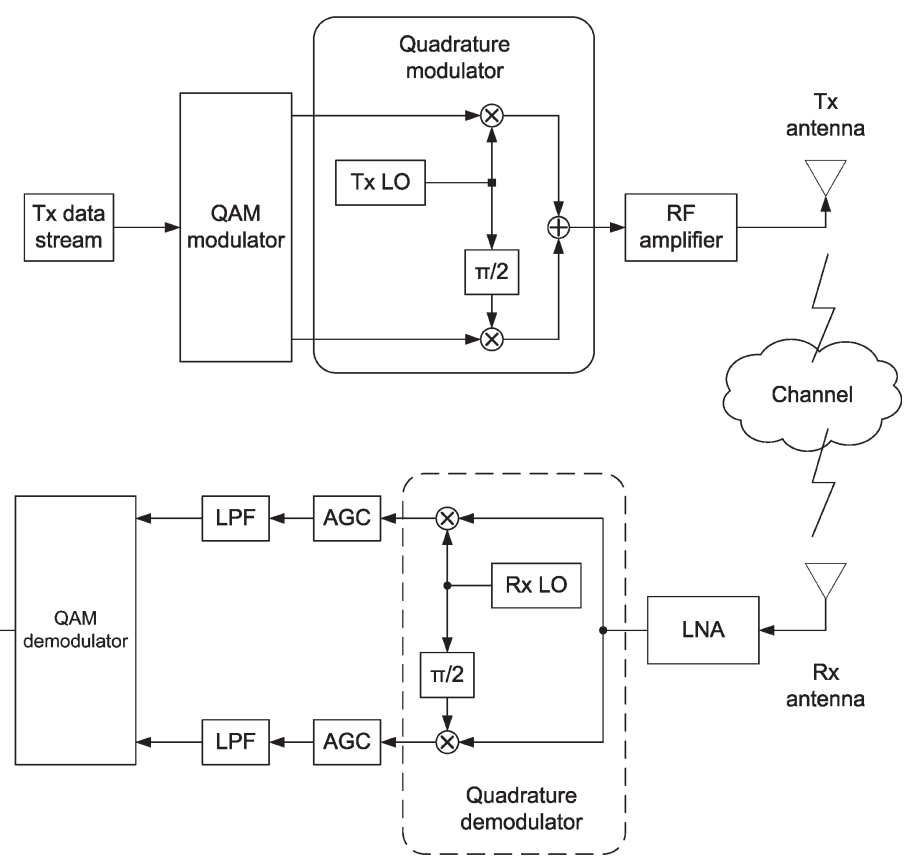

Fig. 1. System model for an $M$-QAM transceiver.

and amplitude imbalances and phase noise in both transmitter and receiver LOs. With this model, a closed-form analytic expression of the EVM is derived, which is more complete compared to the model given in [11] due to the amendment of phase and amplitude imbalances. Moreover, an analytical expression of the SER is obtained in the presence of the imbalances and phase noise. This integration expression of the SER has no closed-form solution and can only be numerically calculated by the finite-element method, which is still more efficient compared to the commonly used Monte-Carlo-based simulation approaches. Thus, the proposed QAM imperfection analysis model provides an efficient means for system engineers to analyze the wireless transceiver performance and specify the transceiver specifications. It can also help the circuit designers as a guide to tradeoffs in designing RFIC transceivers.

This paper is organized as follows. Section II establishes a system model of an $M$-QAM transceiver link with phase and amplitude imbalances, dc offsets, and phase synchronization offset. Section III develops an analytic approach for the EVM expression. Section IV derives the integration expression of the SER. The phase noise is then added in the system model in Section V, and the expressions of the EVM and the SER are obtained in the presence of the phase noise. Section VI provides some calculation results based on the closed-form expressions that are compared with the simulation results. It also gives some analysis and discussions on the effects of imbalances and phase noise on $M$-QAM systems. Finally, conclusions are drawn in Section VII.

\section{SySTEM MOdEL}

This paper focuses on a one-directional physical link between the coder in the transmitter ( $\mathrm{Tx}$ ) and the decoder in the receiver (Rx). As shown in Fig. 1, a full system model is composed of a QAM modulator and demodulator, a quadrature modulator and demodulator, an RF amplifier, a low noise amplifier (LNA), an automatic gain control (AGC), low-pass filters (LPFs), and antennas. The input and output of this system model are data streams to and from the coder-decoder (codec). The SER will be analytically derived and used as the major parameter to assess the performance of this system model.

In a QAM modulator, the transmitted symbols are mapped to a constellation with $M$ signal points in a lattice, which are different in both the phase and amplitude. Then, those discrete signal points excite a shaping filter to generate the baseband signal in two channels that are called in-phase channel (I channel) and quadrature-phase channel (Q channel). The QAM demodulator performs the reversed function, i.e., the received twochannel baseband signals are filtered by a matching filter and sampled to get the received signal points, which are then decided to be received symbols.

The quadrature modulator upconverts the two-channel baseband signals to an RF band. The carriers for both channels are generated by a transmitter LO generator, which is normally a PLL with quadrature outputs. The baseband I-Q signals are upconverted using an image-rejection mixer with the singlesideband output. The quadrature demodulator downconverts the received RF signal to $\mathrm{I}-\mathrm{Q}$ baseband signals by mixing it with the quadrature carriers generated by an Rx LO generator. The Tx and Rx LOs can be generated with the same PLL synthesizer if the uplink and downlink operate at the same frequency. The two LPFs in the receiver path provide the baseband filtering. The RF amplifier, the LNA, and the AGCs are used to adjust the received and transmitted signal powers.

In the implementations of this system, several imperfections are introduced from different components and should be considered. Transceiver building blocks such as the LNA, the AGC, and the RF amplifier can introduce noise and nonlinearity. The amplitude imbalance, phase imbalance, dc offset, and phase noise are presented in the LOs in the quadrature modulator 


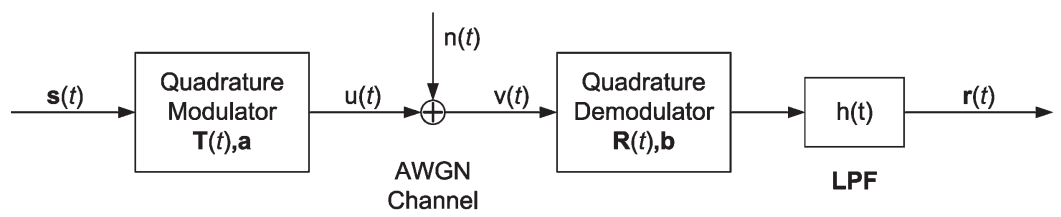

Fig. 2. Equivalent system model.

and demodulator. In wireless transceiver RFIC designs, the commonly used techniques for quadrature signal generation can be summarized as follows: 1) A divided-by-two frequency divider following the VCO running at double the frequency. This approach generally shows poor quadrature accuracy, as it requires $50 \%$ duty cycle $\mathrm{VCO}$ output. Therefore, there will be a non-negligible phase imbalance at the divider-by-two output. 2) A VCO followed by a passive polyphase $R C$ complex filter. An integrated $R C-C R$ network will suffer from process spread on the $R C$ time constant, resulting in the amplitude imbalance between the quadrature outputs. Furthermore, the typical $R C$ phase-shift network will also load the RF oscillator. A buffer after the oscillator to relieve this loading will be power hungry and may attenuate this signal. The polyphase network is normally of narrow band with poor quadrature amplitude and phase accuracy. 3) Two VCOs forced to run in quadrature by using transformer coupling. This technique provides wideband quadrature accuracy with a tradeoff of large silicon area for transformer designs. 4) Two VCOs forced to run in quadrature by using coupling transistors. This technique provides wideband quadrature accuracy with a tradeoff of phase noise. The aforementioned two quadrature VCO designs suffer from large power consumption to operate two VCOs, as well as non-negligible phase noise and phase/amplitude imbalances. Therefore, phase and amplitude imbalances have to be considered in transceiver RFIC designs for QAM applications. At the receiver, the nonideal synchronizations of the frequency and phase of carrier and sample timing will also degrade the system performance. LO synchronization is a critical requirement for multiple-input-multiple-output transceiver RFIC designs [13]. For a real system, the channel may have the features of frequency-selective, time-varying, and doubly selective fading for different application environments.

This paper will discuss amplitude imbalance, phase imbalance, dc offset, and phase noise. Other imperfections are neglected but shown here to give a full picture of the system consideration. To simplify the problem, an additive white Gaussian noise (AWGN) channel is used as the channel model in the analysis.

Under those considerations, the equivalent system model in vector presentation is shown in Fig. 2. The $\mathbf{s}(t)=$ $\left[s_{i}(t), s_{q}(t)\right]^{\mathrm{T}}$ presents the shaped QAM output, where the superscript $\mathrm{T}$ denotes the transpose operation. The quadrature modulator imperfection is modeled by a vector $\mathbf{a}=\left[a_{i}, a_{q}\right]^{\mathrm{T}}$, which represents the dc offset and a $1 \times 2$ matrix $\mathbf{T}(t)$ as

$$
\mathbf{T}(t)=2[k \cos (\omega t), \sin (\omega t+\phi)]
$$

where $\omega$ is the carrier angular frequency, $k$ presents the amplitude imbalance, which has a value of around 1 , and $\varphi$ presents the phase imbalance, whose magnitude is much smaller than $\pi / 2$. The constant 2 is inserted to keep the gain of the transceiver link to be one. Therefore, the output signal from the quadrature modulator is a time-varying scalar expressed as

$$
u(t)=\mathbf{T}(t)[\mathbf{s}(t)+\mathbf{a}] .
$$

Similar to the modulator, the imperfection of the quadrature demodulator is modeled by a vector $\mathbf{b}=\left[b_{i}, b_{q}\right]^{\mathrm{T}}$ and a $2 \times 1$ matrix $\mathbf{R}(t)$ as

$$
\mathbf{R}(t)=[l \cos (\omega t+\alpha), \sin (\omega t+\alpha+\gamma)]^{\mathrm{T}}
$$

where $\alpha$ presents a constant phase difference between the transmitter LO and the receiver $\mathrm{LO}$, which is introduced by the nonideal synchronization of the carrier, $l$ presents the amplitude imbalance, and $\gamma$ presents the phase imbalance. Let $n(t)$ denote AWGN with a power single-side spectrum density (PSD) of $N_{0}$. Using all aforementioned expressions, the output signal $\mathbf{r}(t)$ can be expressed as

$$
\begin{aligned}
\mathbf{r}(t) & =\operatorname{LPF}\langle\mathbf{R}(t)\{\mathbf{T}(t)[\mathbf{s}(t)+\mathbf{a}]+n(t)\}+\mathbf{b}\rangle \\
& =\operatorname{LPF}\langle\mathbf{R}(t) \mathbf{T}(t) \mathbf{s}(t)+\mathbf{R}(t) \mathbf{T}(t) \mathbf{a}+\mathbf{b}+\mathbf{R}(t) n(t)\rangle
\end{aligned}
$$

where $\operatorname{LPF}\langle\rangle$ presents the function of the LPF. Considering that the signal bandwidth is smaller than the bandwidth of the LPF and the RF carrier frequency is out of the passband of the baseband LPF, (4) can be rewritten as

$$
\mathbf{r}(t)=\mathbf{H s}(t)+\mathbf{c}+\mathbf{n}_{r}(t)
$$

with the output constant offset given as

$$
\mathbf{c}=\mathbf{H a}+\mathbf{b}
$$

the channel matrix given as in (7), shown at the bottom of the next page, and the received channel noise given as

$$
\mathbf{n}_{r}(t)=\operatorname{LPF}\langle\mathbf{R}(t) n(t)\rangle .
$$

In (7), it is observed that the channel matrix $\mathbf{H}$ is no longer dependent on time, because the high-frequency components are suppressed by the LPF. The received noise $\mathbf{n}_{r}(t)$ is still a Gaussian noise, because it comes from a linear transform of the Gaussian channel noise. It has zero mean, and its covariance matrix is given by (as detailed in the Appendix)

$$
\mathbf{C}_{\mathbf{n}_{r} \mathbf{n}_{r}}=\frac{N_{0} B}{4}\left[\begin{array}{cc}
l^{2} & l \sin (\gamma) \\
l \sin (\gamma) & 1
\end{array}\right]
$$

where $B$ presents the bandwidth of the LPF. Thus, the presence of the receiver phase imbalance results in a correlated noise in I and Q channels. 


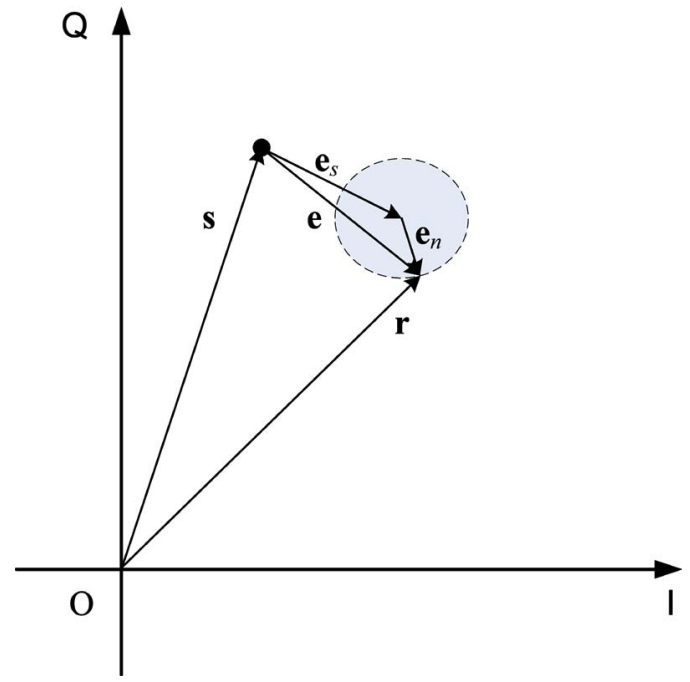

Fig. 3. Error vector.

Assuming an ideal synchronization of the sampling time, the received signal samples are then expressed as

$$
\mathbf{r}(n)=\mathbf{H s}(n)+\mathbf{c}+\mathbf{n}_{r}(n)
$$

where the channel matrix $\mathbf{H}$ is the same as that expressed in (7), the noise $\mathbf{n}_{r}(n)$ has the same covariance matrix as that given in (9), and $\boldsymbol{\nu}(n)=\boldsymbol{\nu}\left(n T_{s}\right), \boldsymbol{\nu}=\mathbf{r}, \mathbf{s}, \mathbf{n}_{r}$, are sampled signals with the symbol period of $T_{s}$.

\section{EVM CALCULATION}

In the communication system, the modulation accuracy is quantified by the EVM, which is defined as the root-meansquare error between the sent and received signal vectors. For the system model discussed in this paper, the EVM can be obtained from (10).

First, the error vector is the difference between the transmitted and received signal vectors, namely

$\mathbf{e}(n)=\mathbf{r}(n)-\mathbf{s}(n)=(\mathbf{H}-\mathbf{I}) \mathbf{s}(n)+\mathbf{c}+\mathbf{n}_{r}(n)=\mathbf{e}_{s}(n)+\mathbf{e}_{n}(n)$.

The aforementioned error vector consists of two components: one, which is denoted by $\mathbf{e}_{s}(n)=(\mathbf{H}-\mathbf{I}) \mathbf{s}(n)+\mathbf{c}$, is related to the signal vector and is determined by a given symbol, and the other, which is denoted by $\mathbf{e}_{n}(n)=\mathbf{n}_{r}(n)$, comes from channel random noise with a Gaussian distribution. Fig. 3 illustrates the definition of the error vector and its two components.
From the error vector expressed in (11), the EVM can be found out as

$$
\begin{aligned}
\mathrm{EVM}^{2}= & \sigma_{\mathbf{e}}^{2}=E\left[\mathbf{e}^{\mathrm{H}}(n) \mathbf{e}(n)\right]=E\left[\mathbf{e}^{\mathrm{T}}(n) \mathbf{e}(n)\right] \\
=E[ & \left((\mathbf{H}-\mathbf{I}) \mathbf{s}(n)+\mathbf{c}+\mathbf{n}_{r}(n)\right)^{\mathrm{T}} \\
& \left.\times\left((\mathbf{H}-\mathbf{I}) \mathbf{s}(n)+\mathbf{c}+\mathbf{n}_{r}(n)\right)\right]
\end{aligned}
$$

where the superscript $\mathrm{H}$ denotes the Hermitian operator. Because all vectors and matrices in the approach are real, the Hermitian operator is then equivalent to the transpose operator that will be used in the following derivations. Under the assumptions that the transmitted signal vectors have zero mean and are independent of noise and that the I and Q components of the signal vectors are independent, (12) can be simplified as

$$
\begin{aligned}
E V M^{2}=E\left[\mathbf{s}^{\mathrm{T}}(n)(\mathbf{H}-\mathbf{I})^{\mathrm{T}}(\mathbf{H}-\mathbf{I}) \mathbf{s}(n)\right] \\
+\mathbf{c}^{\mathrm{T}} \mathbf{c}+E\left[\mathbf{n}_{r}(n)^{\mathrm{T}} \mathbf{n}_{r}(n)\right] .
\end{aligned}
$$

Based on the aforementioned assumptions, the covariance matrix of signal is obtained as

$$
\mathbf{c}_{\mathbf{s}(n) \mathbf{s}(n)}=\frac{E_{\mathrm{S}} R}{2} \mathbf{I}
$$

where $E_{s}$ presents the average symbol energy, $R$ presents the symbol rate, and I presents a unit matrix. Therefore, with (7), the first term in the right hand of (13) can be expressed as

$$
\begin{aligned}
& E\left[\mathbf{s}^{\mathrm{T}}(n)(\mathbf{H}-\mathbf{I})^{\mathrm{T}}(\mathbf{H}-\mathbf{I}) \mathbf{s}(n)\right] \\
& =\frac{E_{\mathrm{s}} R}{2} \operatorname{Tr}\left((\mathbf{H}-\mathbf{I})^{\mathrm{T}}(\mathbf{H}-\mathbf{I})\right) \\
& =\frac{E_{\mathrm{s}} R}{2}\left[\operatorname{Tr}\left(\mathbf{H}^{\mathrm{T}} \mathbf{H}\right)-2 \operatorname{Tr}(\mathbf{H})+2\right] \\
& =\frac{E_{s} R}{2}\left[\left(k^{2} l^{2} \cos ^{2}(\alpha)+k^{2} \sin ^{2}(\phi-\alpha)+l^{2} \sin ^{2}(\alpha+\gamma)\right.\right. \\
& \left.\quad+\cos ^{2}(\alpha+\gamma-\phi)\right) \\
& \quad-2(k l \cos (\alpha)+\cos (\alpha+\gamma-\phi))+2] .
\end{aligned}
$$

From (9), it can be obtained that

$$
E\left[\mathbf{n}_{r}(n)^{\mathrm{T}} \mathbf{n}_{r}(n)\right]=\frac{N_{0} B\left(l^{2}+1\right)}{4} .
$$

$$
\begin{aligned}
\mathbf{H} & =\operatorname{LPF}\langle\mathbf{R}(t) \mathbf{T}(t)\rangle \\
& =\operatorname{LPF}\left\langle 2[l \cos (\omega t+\alpha), \sin (\omega t+\alpha+\gamma)]^{\mathrm{T}}[k \cos (\omega t), \sin (\omega t+\phi)]\right\rangle \\
& =\operatorname{LPF}\left\langle 2\left[\begin{array}{cc}
k l \cos (\omega t+\alpha) \cos (\omega t) & l \cos (\omega t+\alpha) \sin (\omega t+\phi) \\
k \sin (\omega t+\alpha+\gamma) \cos (\omega t) & \sin (\omega t+\alpha+\gamma) \sin (\omega t+\phi)
\end{array}\right]\right\rangle \\
& =\left[\begin{array}{cc}
k l \cos (\alpha) & l \sin (\phi-\alpha) \\
k \sin (\alpha+\gamma) & \cos (\alpha+\gamma-\phi)
\end{array}\right]
\end{aligned}
$$




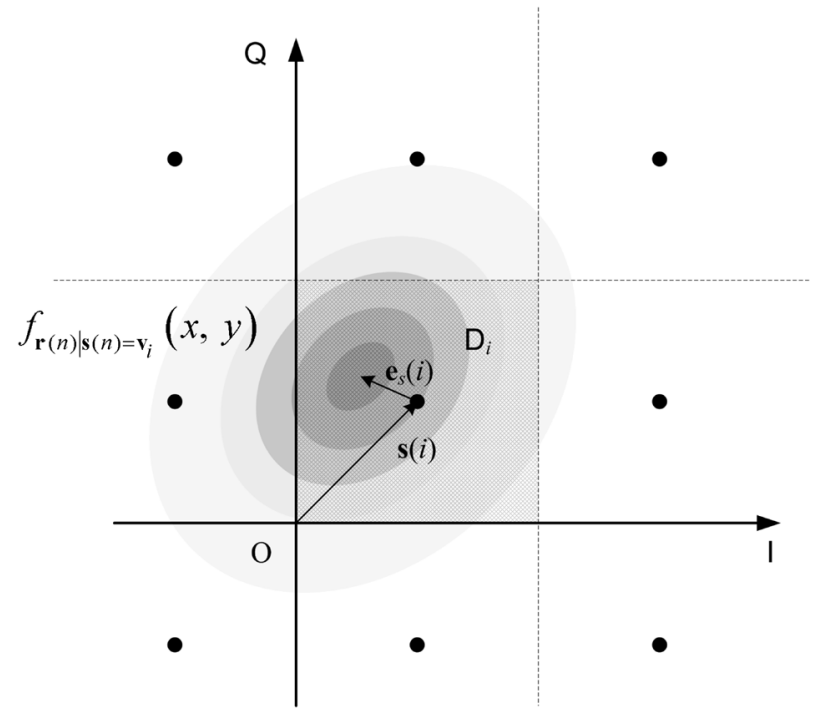

Fig. 4. PDF of the received vector and the decision zone.

Moreover, from (6)

$$
\mathbf{c}^{\mathrm{T}} \mathbf{c}=\mathbf{a}^{\mathrm{T}} \mathbf{H}^{\mathrm{T}} \mathbf{H a}+\mathbf{a}^{\mathrm{T}} \mathbf{H}^{\mathrm{T}} \mathbf{b}+\mathbf{b}^{\mathrm{T}} \mathbf{H a}+\mathbf{b}^{\mathrm{T}} \mathbf{b} .
$$

By substituting (15), -(17) into (13), a detailed expression of the EVM is obtained but will not be listed here for conciseness.

In practice, the EVM is usually normalized by the signal power $P=R E_{s}$, and by assuming that the symbol rate equals to the pass bandwidth of the LPF, i.e., $R=B$, the normalized EVM is thus obtained as

$\mathrm{EVM}_{\text {normalized }}$

$$
=\sqrt{\frac{1}{2} \operatorname{Tr}\left(\mathbf{H}^{\mathrm{T}} \mathbf{H}\right)-\operatorname{Tr}(\mathbf{H})+1+\frac{\left(l^{2}+1\right)}{4 E_{s} / N_{0}}+\frac{\mathbf{c}^{\mathrm{T}} \mathbf{c}}{P} .}
$$

In the radical sign, $\operatorname{Tr}\left(\mathbf{H}^{\mathrm{T}} \mathbf{H}\right) / 2-\operatorname{Tr}(\mathbf{H})+1$ represents the contribution of the phase and amplitude imbalances to the EVM, $\left(l^{2}+1\right) /\left(4 E_{s} / N_{0}\right)$ represents the contribution of the channel noise, and $\mathbf{c}^{\mathrm{T}} \mathbf{c} / P$ represents the contribution of the constant offsets. By using (18), the effects of the transceiver imbalances, channel noise, and modulation offsets on the EVM can further be analyzed.

\section{SER CALCUlation}

Considering an $M$-QAM, all possible symbols are defined as an alphabet of $\mathbb{S}=\left\{s_{1}, s_{2}, \ldots s_{N}\right\}$. Furthermore, the corresponding modulated vectors are defined as a vector alphabet of $\mathbb{V}=\left\{\mathbf{v}_{1}, \mathbf{v}_{2}, \ldots \mathbf{v}_{N}\right\}$. Recalling (10) and (11), for a given transmitted signal vector $\mathbf{s}(n) \in \mathbb{V}$, the received vector is expressed as

$$
\mathbf{r}(n)=\mathbf{s}(n)+\mathbf{e}_{s}(n)+\mathbf{e}_{n}(n) .
$$

As illustrated in Fig. 4, the received vector has a deterministic component $\mathbf{s}(n)+\mathbf{e}_{s}(n)$ and a random component $\mathbf{e}_{n}(n)$. With the covariance matrix obtained in (9), the Gaussian noise $\mathbf{e}_{n}(n)$ has a joint normal probability density function (pdf) that is expressed as

$$
\begin{aligned}
f_{\mathbf{e}_{n}}(x, y) & =N_{2}\left(0,0, N_{0} B l^{2} / 4 N_{0} B / 4, \sin (\gamma)\right) \\
= & A \exp \left\{-\frac{2}{N_{0} B\left(1-\sin ^{2}(\gamma)\right)}\right. \\
& \left.\times\left[\frac{x^{2}}{l^{2}}-2 \sin (\gamma) \frac{x y}{l}+y^{2}\right]\right\} \\
A & =\frac{2}{\pi N_{0} B l \sqrt{1-\sin ^{2}(\gamma)}}
\end{aligned}
$$

where $N_{2}()$ denotes a 2-D joint normal distribution pdf, and $x$ and $y$ denote the $\mathrm{I}$ and $\mathrm{Q}$ components of received noise vector that are zero-mean random variables with variances and correlation given by the covariance matrix in (9). In Fig. 4, the concentric ellipses in different gray colors illustrate the pdf of the noise. Because the two channels of the noise are correlated to each other, the contours of the pdf are not circles but tilt ellipses. Moreover, because this noise term is independent of the signal, the received noise components have an identical pdf and do not relate to different transmitted signal vectors.

From (19) and (20), the conditional pdf of the received vector can be obtained as

$$
\begin{aligned}
& f_{\mathbf{r}(n) \mid \mathbf{s}(n)}(x, y)= f_{\mathbf{e}_{n}}\left(x-\bar{r}_{x}(n), y-\bar{r}_{y}(n)\right) \\
&= N_{2}\left(\bar{r}_{x}(n), \bar{r}_{y}(n), N_{0} B l^{2} / 4 N_{0} B / 4 \sin (\gamma)\right) \\
&= A \exp \left\{-\frac{4}{N_{0} B\left(1-\sin ^{2}(\gamma)\right)}\right. \\
& \times\left[\frac{\left(x-\bar{r}_{x}(n)\right)^{2}}{l^{2}}-2 \sin (\gamma)\right. \\
& \times \frac{\left(x-\bar{r}_{x}(n)\right)\left(y-\bar{r}_{y}(n)\right)}{l} \\
&\left.\left.+\left(y-\bar{r}_{y}(n)\right)^{2}\right]\right\} \\
& \bar{r}_{x}(n)=I\left\langle\mathbf{s}(n)+\mathbf{e}_{s}(n)\right\rangle \\
& \bar{r}_{y}(n)=Q\left\langle\mathbf{s}(n)+\mathbf{e}_{s}(n)\right\rangle
\end{aligned}
$$

where $I\langle\rangle$ and $Q\langle\rangle$ are the functions to get the I and Q components from a vector, respectively, and $\bar{r}_{x}(i)$ and $\bar{r}_{y}(i)$ are the mean values of the I and Q components of the received vector.

For the system model considered in this paper, under the assumption that the channel and all devices are linear and memoryless, the received QAM modulated vectors are demodulated by hard-decision criteria. Thus, the decision of a received QAM symbol is made by choosing the symbol that minimizes the geometric distance from the received signal vector to the assumed transmitted signal vector in the signal vector alphabet $n$. Each inner constellation point has four nearest neighbors and a square decision zone. The square area shaded by cross lines in Fig. 4 , i.e., $\mathbf{D}_{i}$, shows a decision zone of the transmitted signal vector $\mathbf{v}_{i}$. Therefore, the conditional detection probability is the integral of the conditional pdf of the received sample vector in the decision zone $\mathbf{D}_{i}$, namely

$$
P_{D_{i}}=\iint_{\mathbf{D}_{i}} f_{\mathbf{r}(n) \mid \mathbf{s}(n)=(\xi-\tau)_{i}}(x, y) d x d y .
$$


Furthermore, the conditional error probability is the integration of the conditional pdf of the received sample vector in the area other than the decision domain $\mathbf{D}_{i}$, which is a complement of the conditional detection probability, i.e.,

$$
P_{e_{i}}=1-P_{D_{i}}
$$

In the constellation, the edge points and corner points have three or two nearest neighbors, respectively. Then, the decision zone becomes a one-end-open strip or a quarter plane. The decision zone of the three aforementioned situations can be expressed in a same form as $D_{i}=\left\{x, y \mid x \in\left(a_{i}, b_{i}\right) \mathrm{y} \in\right.$ $\left.\left(c_{i}, d_{i}\right)\right\}$, where the boundaries can be either finite or infinite. By substituting the expression of the decision zone and (22) into (24), the detection probability is expressed as

$$
\begin{aligned}
& P_{D_{i}}=\int_{c_{i}}^{d_{i}} \int_{a_{i}}^{b_{i}} A \exp \left\{-\frac{4}{N_{0} B\left(1-\sin ^{2}(\gamma)\right)}\right. \\
& \times\left[\frac{\left(x-\bar{r}_{x}(n)\right)^{2}}{l^{2}}-2 \sin (\gamma)\right. \\
& \times \frac{\left(x-\bar{r}_{x}(n)\right)\left(y-\bar{r}_{y}(n)\right)}{l} \\
& \left.\left.+\left(y-\bar{r}_{y}(n)\right)^{2}\right]\right\} d x d y .
\end{aligned}
$$

Because there is a mixture term of $x$ and $y$ in the integrand, the aforementioned integral does not have a closed-form solution and can only be calculated by a numerical integral method. To calculate the total detection probability for all possible transmitted symbol, this integral needs to be calculated for each symbol. An efficient method to do the numerical integral is derived by converting (26) into

$$
P_{D_{i}}=\int_{c_{i}-\bar{r}_{y}(n)}^{d_{i}-\bar{r}_{y}(n)} \int_{a_{i}-\bar{r}_{x}(n)}^{b_{i}-\bar{r}_{x}(n)} N_{2}\left(0,0, N_{0} B l^{2} / 4, N_{0} B / 4, \sin (\gamma)\right) d x d y .
$$

Therefore, the integrand is no longer related to the symbols. The numerical integral then can be estimated by a finiteelement method, i.e., calculating the integrand values in grids and accumulating grids in different integral fields.

For 32-QAM and 128-QAM, the decision zone of corner points will have a sloped boundary, which will make the calculation of the SER more complicated. The SER calculation for 32-QAM and 128-QAM is not given here, yet the results can be obtained by slightly extending the work of this paper.
Based on a basic assumption that each symbol in $n$ has the same probability to be sent, the total SER is obtained by averaging the conditional detection error probability of each symbol as

$$
P_{e}=\frac{1}{N} \sum_{i=0}^{N-1} P_{e_{i}}
$$

Equation (28) gives an analytical expression of the SER for an $M$-QAM system in the presence of transceiver imbalances and channel noise.

\section{Phase Noise Consideration}

In the aforementioned sections, the phase noise has not been taken into account, and all phase variables presented in the equations are deterministic. For a more realistic consideration of the transceiver system discussed in this paper, the LO signal in both the transmitter and the receiver is not an ideal tone but a carrier with phase noise presented in the frequency domain or a jittered signal presented in the time domain. The phase noise then results in both random phase imbalances in quadrature modulators and random phase difference between two LOs.

The detailed phase analysis is beyond the scope of this paper. Instead, we will simply approximate the phase noise as a zeromean Gaussian noise in phase representation whose variance is defined as the LO mean square phase error that can be obtained from other works [1], [8].

\section{A. EVM Calculation}

All approaches in the aforementioned sections are formally valid, except that the phase variables should be understood as random variables. Therefore, the phase difference $\alpha$ is updated as

$$
\alpha=\alpha_{d}+\alpha_{r}, \quad \alpha_{r} \sim N\left(0, \alpha_{\mathrm{rms}}^{2}\right)
$$

where $\alpha_{d}$ denotes a constant phase difference, and $\alpha_{r}$ is a Gaussian random variable that represents the phase noise.

Substituting (29) to (7), we have

$$
\mathbf{H}=\left[\begin{array}{cc}
k l \cos \left(\alpha_{d}+\alpha_{r}\right) & l \sin \left(\phi-\alpha_{d}-\alpha_{r}\right) \\
k \sin \left(\alpha_{d}+\alpha_{r}+\gamma\right) & \cos \left(\alpha_{d}+\alpha_{r}+\gamma-\phi\right)
\end{array}\right] .
$$

Under the assumption that $\alpha_{\mathrm{rms}} \ll 1$, the aforementioned equation can be simplified as in (31), shown at the bottom of the page, where $\mathbf{H}_{d}$ has the same form as $\mathbf{H}$ in (7), and $\alpha_{r}$

$$
\begin{aligned}
\mathbf{H} & =\left[\begin{array}{cc}
k l \cos \left(\alpha_{d}\right)-k l \alpha_{r} \sin \left(\alpha_{d}\right) & l \sin \left(\phi-\alpha_{d}\right)-l \alpha_{r} \cos \left(\phi-\alpha_{d}\right) \\
k \sin \left(\alpha_{d}+\gamma\right)+k \alpha_{r} \cos \left(\alpha_{d}+\gamma\right) & \cos \left(\alpha_{d}+\gamma-\phi\right)-\alpha_{r} \sin \left(\alpha_{d}+\gamma-\phi\right)
\end{array}\right] \\
& =\left[\begin{array}{cc}
k l \cos \left(\alpha_{d}\right) & l \sin \left(\phi-\alpha_{d}\right) \\
k \sin \left(\alpha_{d}+\gamma\right) & \cos \left(\alpha_{d}+\gamma-\phi\right)
\end{array}\right]+\alpha_{r}\left[\begin{array}{cc}
-k l \sin \left(\alpha_{d}\right) & -l \cos \left(\phi-\alpha_{d}\right) \\
k \cos \left(\alpha_{d}+\gamma\right) & -\sin \left(\alpha_{d}+\gamma-\phi\right)
\end{array}\right] \\
& \triangleq \mathbf{H}_{d}+\alpha_{r} \mathbf{H}_{r}
\end{aligned}
$$




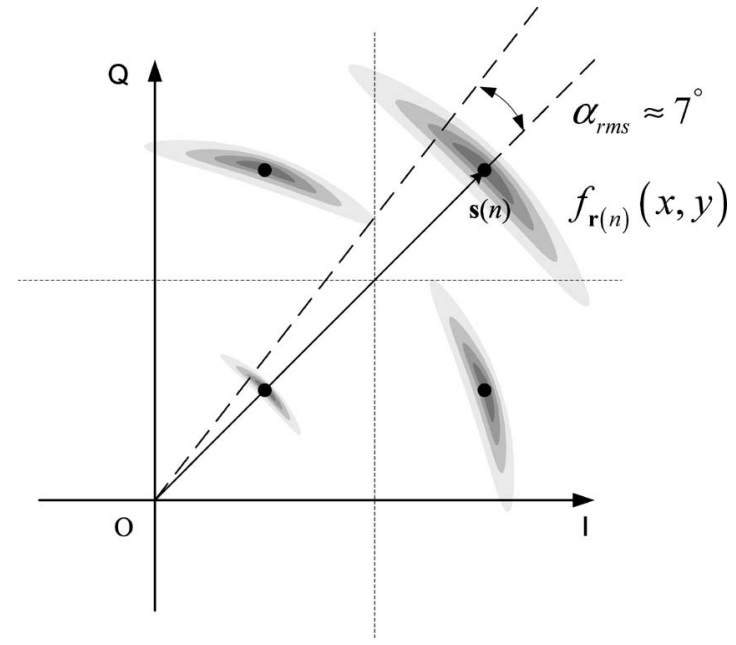

Fig. 5. PDF of the phase noise for different constellation points.

is factored out of the matrix and multiplied by a deterministic matrix $H_{r}$. Then, (10) becomes

$$
\mathbf{r}(n)=\mathbf{H}_{d} \mathbf{s}(n)+\alpha_{r} \mathbf{H}_{r}(\mathbf{s}(n)+\mathbf{a})+\mathbf{c}+\mathbf{n}_{r}(n)
$$

where $\mathbf{c}=\mathbf{H}_{d} \mathbf{a}+\mathbf{b}$. Comparing (32) with (10), a new term, i.e., $\mathbf{n}_{p}(n) \triangleq \alpha_{r} \mathbf{H}_{r}(\mathbf{s}(n)+\mathbf{a})$, appears, which represents the effect of the LO phase noise on the received signal. Unlike the channel noise, which is additive, the phase noise is multiplied with the signal. Furthermore, observing the matrix $\mathbf{H}_{r}$, if the phase imbalances and phase difference are small, it approximates to an anti-identity matrix. Then, the phase noise term $\mathbf{n}_{p}(n)$ is approximately perpendicular to the signal vector, which means that the error due to the phase noise is mostly on the phase and not on the amplitude. Fig. 5 illustrates the pdf of the received signal vector in the presence of phase noise with $\alpha_{\text {rms }} \approx 7^{\circ}$, which intuitively shows that the constellation point with a larger amplitude is influenced more by the phase noise.

Considering that the phase noise is independent of the signal, the covariance matrix of $\mathbf{n}_{p}(n)$ is obtained as

$$
\begin{aligned}
\mathbf{C}_{\mathbf{n}_{p} \mathbf{n}_{p}} & =E\left[\alpha_{r} \mathbf{H}_{r}(\mathbf{s}(n)+\mathbf{a})\left(\alpha_{r} \mathbf{H}_{r}(\mathbf{s}(n)+\mathbf{a})\right)^{\mathrm{T}}\right] \\
& =E\left[\alpha_{r}^{2}\right] \mathbf{H}_{r} E\left[\mathbf{s}(n) \mathbf{s}^{\mathrm{T}}(n)\right] \mathbf{H}_{r}^{\mathrm{T}}+E\left[\alpha_{r}^{2}\right] \mathbf{H}_{r} \mathbf{a a}^{\mathrm{T}} \mathbf{H}_{r}^{\mathrm{T}} \\
& =\frac{E_{\mathrm{s}} R \alpha_{\mathrm{rms}}^{2}}{2} \mathbf{H}_{r} \mathbf{H}_{r}^{T}+\alpha_{\mathrm{rms}}^{2} \mathbf{H}_{r} \mathbf{a a}^{\mathrm{T}} \mathbf{H}_{r}^{\mathrm{T}} .
\end{aligned}
$$

Note that, in the phase noise term in (32), the $\mathbf{s}(n), \alpha_{r}$, and $\mathbf{n}_{r}(n)$ are of zero mean and independent of each other, and the EVM in (13) becomes

$$
\begin{aligned}
\mathrm{EVM}^{2}=E\left[\mathbf{s}^{\mathrm{T}}(n)\left(\mathbf{H}_{d}+\alpha_{r} \mathbf{H}_{r}-\mathbf{I}\right)^{\mathrm{T}}\left(\mathbf{H}_{d}+\alpha_{r} \mathbf{H}_{r}-\mathbf{I}\right) \mathbf{s}(n)\right] \\
+\alpha_{\mathrm{rms}}^{2} \mathbf{a}^{\mathrm{T}} \mathbf{H}_{r}^{\mathrm{T}} \mathbf{H}_{r} \mathbf{a}+\mathbf{c}^{\mathrm{T}} \mathbf{c}+\frac{1}{4} N_{0} B\left(l^{2}+1\right) .
\end{aligned}
$$

Since the phase noise is of zero mean and independent of the signal, substituting (31) in the first term in the right-hand side of (34) leads to the following expression:

$$
\begin{aligned}
& E\left[\mathbf{s}^{\mathrm{T}}(n)\left(\mathbf{H}_{d}+\alpha_{r} \mathbf{H}_{r}-\mathbf{I}\right)^{\mathrm{T}}\left(\mathbf{H}_{d}+\alpha_{r} \mathbf{H}_{r}-\mathbf{I}\right) \mathbf{s}(n)\right] \\
&= E\left[\mathbf{s}^{\mathrm{T}}(n)\left(\mathbf{H}_{d}-\mathbf{I}\right)^{\mathrm{T}}\left(\mathbf{H}_{d}-\mathbf{I}\right) \mathbf{s}(n)\right] \\
&+E\left[\mathbf{s}^{\mathrm{T}}(n) \alpha_{r} \mathbf{H}_{r}^{\mathrm{T}}\left(\mathbf{H}_{d}-\mathbf{I}\right) \mathbf{s}(n)\right] \\
&+E\left[\mathbf{s}^{\mathrm{T}}(n) \alpha_{r}\left(\mathbf{H}_{d}-\mathbf{I}\right)^{\mathrm{T}} \mathbf{H}_{r} \mathbf{s}(n)\right] \\
&+E\left[\mathbf{s}^{\mathrm{T}}(n) \alpha_{r}^{2} \mathbf{H}_{r}^{\mathrm{T}} \mathbf{H}_{r} \mathbf{s}(n)\right] \\
&= E\left[\mathbf{s}^{\mathrm{T}}(n)\left(\mathbf{H}_{d}-\mathbf{I}\right)^{\mathrm{T}}\left(\mathbf{H}_{d}-(\xi-\tau) \mathbf{I}\right) \mathbf{s}(n)\right] \\
&+E\left[\alpha_{r}^{2}\right] E\left[\mathbf{s}^{\mathrm{T}}(n) \mathbf{H}_{r}^{\mathrm{T}} \mathbf{H}_{r} \mathbf{s}(n)\right] \\
&= \frac{E_{\mathrm{s}} R}{2}\left[\operatorname{Tr}\left(\mathbf{H}_{d}^{\mathrm{T}} \mathbf{H}_{d}\right)-2 \operatorname{Tr}\left(\mathbf{H}_{d}\right)+2\right] \\
&+\frac{E_{\mathrm{s}} R \alpha_{\mathrm{rms}}^{2}}{2} \operatorname{Tr}\left(\mathbf{H}_{r}^{\mathrm{T}} \mathbf{H}_{r}\right) .
\end{aligned}
$$

Therefore, the normalized EVM in (18) becomes that in (36), shown at the bottom of the page.

Comparing with (18), in the radical sign, $\operatorname{Tr}\left(\mathbf{H}_{d}^{\mathrm{T}} \mathbf{H}_{d}\right) / 2-$ $\operatorname{Tr}\left(\mathbf{H}_{d}\right)+1$ represents the contribution of the phase and amplitude imbalances to the EVM and keeps the same form, $\left(l^{2}+1\right) /\left(4 E_{s} / N_{0}\right)$ represents the contribution of the channel noise and also keeps the form, $\left(\alpha_{\mathrm{rms}}^{2} \mathbf{a}^{\mathrm{T}} \mathbf{H}_{r}^{\mathrm{T}} \mathbf{H}_{r} \mathbf{a}+\mathbf{c}^{\mathrm{T}} \mathbf{c}\right) / P$ represents the contribution of the constant offsets, which increased by a term related to phase noise, and a new component, i.e., $\alpha_{\mathrm{rms}}^{2} \operatorname{Tr}\left(\mathbf{H}_{r}^{\mathrm{T}} \mathbf{H}_{r}\right) / 2$, appears to represent the increased contribution from phase noise to the EVM.

\section{B. SER Calculation}

In the presence of phase noise, the expression of the error of the received signal becomes

$$
\mathbf{r}(n)=\mathbf{s}(n)+\mathbf{e}_{s}(n)+\mathbf{e}_{p}(n)+\mathbf{e}_{n}(n)
$$

where $\mathbf{e}_{s}(n)$ becomes $\left(\mathbf{H}_{d}-\mathbf{I}\right) \mathbf{s}(n)+\mathbf{c}$, and $\mathbf{e}_{p}(n)=$ $\alpha_{r} \mathbf{H}_{r}(\mathbf{s}(n)+\mathbf{a})$ represents the phase noise effect. Since both the channel noise and phase noise are Gaussian, the sum of them, i.e., $\mathbf{e}(n)=\mathbf{e}_{p}(n)+\mathbf{e}_{n}(n)$, is still Gaussian. As shown in Fig. 5, unlike the channel noise $\mathbf{e}_{n}(i)$, which is independent of the signal and has an identical pdf for different symbols, the phase noise term $\mathbf{e}_{p}(i)$ is dependent on the signal. Thus, it has different pdf's for different symbols. Therefore, giving a transmitted symbol, the conditional covariance matrix of $\mathbf{e}_{p}(i)+\mathbf{e}_{n}(i)$ is obtained as

$$
\begin{aligned}
\mathbf{c}_{\mathbf{e e} \mid \mathbf{s}(n)} & =E\left[\alpha_{r} \mathbf{H}_{r}(\mathbf{s}(n)+\mathbf{a})\left(\alpha_{r} \mathbf{H}_{r}(\mathbf{s}(n)+\mathbf{a})\right)^{\mathrm{T}}\right]+\mathbf{C}_{\mathbf{n}_{r} \mathbf{n}_{r}} \\
& =\alpha_{\mathrm{rms}}^{2} \mathbf{H}_{r}(\mathbf{s}(n)+\mathbf{a})(\mathbf{s}(n)+\mathbf{a})^{\mathrm{T}} \mathbf{H}_{r}^{T}+\mathbf{C}_{\mathbf{n}_{r} \mathbf{n}_{r}} \\
& \triangleq\left[\begin{array}{ll}
c_{11} & c_{12} \\
c_{12} & c_{22}
\end{array}\right] .
\end{aligned}
$$

$$
\mathrm{EVM}_{\text {normalized }}=\sqrt{\frac{1}{2} \operatorname{Tr}\left(\mathbf{H}_{d}^{\mathrm{T}} \mathbf{H}_{d}\right)-\operatorname{Tr}\left(\mathbf{H}_{d}\right)+1+\frac{\alpha_{\mathrm{rms}}^{2}}{2} \operatorname{Tr}\left(\mathbf{H}_{r}^{\mathrm{T}} \mathbf{H}_{r}\right)+\frac{\left(l^{2}+1\right)}{4 E_{s} / N_{0}}+\frac{\alpha_{\mathrm{rmm}}^{2} \mathbf{a}^{\mathrm{T}} \mathbf{H}_{r}^{\mathrm{T}} \mathbf{H}_{r} \mathbf{a}+\mathbf{c}^{\mathrm{T}} \mathbf{c}}{P}}
$$


Then, the pdf of the received signal is expressed as

$$
f_{\mathbf{r}(n) \mid \mathbf{s}(n)}(x, y)=N_{2}\left(\bar{r}_{x}(n), \bar{r}_{y}(n), c_{11}, c_{22}, c_{12} / \sqrt{c_{11} c_{22}}\right) .
$$

Following the similar approach used for (24), (25), and (28) in Section IV, the SER can be calculated in the presence of the phase noise as

$$
P_{e}=\frac{1}{N} \sum_{i=0}^{N-1}\left(1-\iint_{\mathbf{D}_{i}} f_{\mathbf{r}(n) \mid \mathbf{s}(n)=(\xi-\tau)_{i}}(x, y) d x d y\right) .
$$

It should be noticed that the simplified approach in (27) is no longer valid. Although the aforementioned integral has to be numerically calculated for each individual constellation point, this theoretical calculation is still much more efficient than a Monte-Carlo-simulation-based method. Moreover, the proposed SER model provides an accurate calculation for an SER less than $10^{-5}$, which is difficult to achieve by using Monte Carlo simulations.

\section{Phase Noise Presented in Phase Imbalance}

As mentioned in the aforementioned section, the phase noise can also yield the randomization of the phase imbalance. Similar to (29), the phase imbalances in the transmitter and receiver can be decomposed to a deterministic part and a random part as

$$
\begin{aligned}
\phi & =\phi_{d}+\phi_{r}, & \phi_{r} & \sim N\left(0, \phi_{\mathrm{rms}}^{2}\right) \\
\gamma & =\gamma_{d}+\gamma_{r}, & \gamma_{r} & \sim N\left(0, \gamma_{\mathrm{rms}}^{2}\right) .
\end{aligned}
$$

Substituting (41) and (42) to (7), respectively, and assuming that $\varphi_{\text {rms }} \ll 1$ and $\gamma_{\text {rms }} \ll 1$, the channel matrix becomes

$$
\begin{aligned}
\mathbf{H} & =\left[\begin{array}{cc}
k l \cos (\alpha) & l \sin \left(\phi_{d}+\phi_{r}-\alpha\right) \\
k \sin (\alpha+\gamma) & \cos \left(\alpha+\gamma-\phi_{d}-\phi_{r}\right)
\end{array}\right] \\
& =\mathbf{H}_{d}+\phi_{r}\left[\begin{array}{cc}
0 & l \cos \left(\phi_{d}-\alpha\right) \\
0 & \sin \left(\alpha+\gamma-\phi_{d}\right)
\end{array}\right] \\
& \triangleq \mathbf{H}_{d}+\phi_{r} \mathbf{H}_{\phi} \\
\mathbf{H} & =\frac{A_{r} A_{t}}{2}\left[\begin{array}{cc}
k l \cos (\alpha) & l \sin (\phi-\alpha) \\
k \sin \left(\alpha+\gamma_{d}+\gamma_{r}\right) & \cos \left(\alpha+\gamma_{d}+\gamma_{r}-\phi\right)
\end{array}\right] \\
& =\mathbf{H}_{d}+\gamma_{r}\left[\begin{array}{cc}
0 & 0 \\
k \cos \left(\alpha+\gamma_{d}\right) & -\sin \left(\alpha+\gamma_{d}-\phi\right)
\end{array}\right] \\
& \triangleq \mathbf{H}_{d}+\gamma_{r} \mathbf{H}_{\phi} .
\end{aligned}
$$

If all phase noise effects on the phase imbalances and phase difference are taken into account, a more sophisticated channel matrix is expressed as

$$
\mathbf{H}=\mathbf{H}_{d}+\alpha_{r} \mathbf{H}_{r}+\phi_{r} \mathbf{H}_{\phi}+\gamma_{r} \mathbf{H}_{\phi} .
$$

Using the channel matrices in (43)-(45), following the aforementioned approach, the calculation of the EVM and SER can be obtained in the presence of random phase imbalance in the transmitter or the receiver or both. It should be noticed that the random phase imbalance in the receiver will affect the received channel noise and change the expression of the covariance matrix in (9). The details of this part of work are very similar to what have been shown in the aforementioned sections and will not be presented in this paper.

\section{Vi. Simulations and Calculations}

In Section 5, the expressions of the EVM (36) and SER (40) are shown, which include all the parameters from the Tx phase and amplitude imbalance, the Tx offset, the Rx phase and amplitude imbalance, the Rx offset, the carrier synchronization phase offset, and the phase noise. A program to calculate the EVM and numerically calculate the SER is built. We also developed a Monte Carlo simulation program to provide the comparison and verification for the analytically calculated results. All results shown below are normalized to the signal power; thus, $B=R=1$ and $E_{s}=1$.

In the model used in this paper, many parameters are taken into account, including the transmitter amplitude imbalance $k$, the phase imbalance $\varphi$, the transmitter offset a, the receiver amplitude imbalance $l$, the phase imbalance $\gamma$, the receiver offset $\mathbf{b}$, the phase difference $\alpha_{d}$, and the phase noise quantified by $\alpha_{\mathrm{rms}}$ and $E_{s} / N_{0}$. Each parameter affects the received signal differently. In Fig. 6, the simulated received signals with different imperfections are shown. Only four points in 64-QAM constellations are shown to clearly illustrate the result.

Figs. 7 and 8 demonstrate two typical situations for 16-QAM and 64-QAM, respectively. As shown in the figures, the results from the analytical calculation are well matched with the results from the Monte Carlo simulation. An observation of the received samples is that the constellation is distorted in a complicated way, which combines the shift, rotation, and shear transformation that come from the many effective parameters aforementioned. Furthermore, the shift and shape of the clouds of received samples are different for different transmitted constellation points. Intuitively, the points with a larger amplitude are affected more by imbalances and phase noise and thus contribute more to the average SER and EVM than the points closer to the origin.

Fig. 9 shows the EVM variation for 16-QAM with the transmitter gain and phase imbalance in the case without channel noise, phase noise, and receiver imbalances. Because of the symmetric characteristic between the transmitter imbalances and receiver imbalances in the channel matrix in (7), the EVM variation with the receiver gain and phase imbalance results in the identical figure. However, the presence of the channel noise will break the symmetry and make the receiver imbalances contribute a little more to the EVM than the transmitter imbalances. The EVM calculation in (18) and (36) also shows that the EVM is not related to the number of the modulation points $N$. Thus, the EVM calculation will give same results for QPSK, 16-QAM, and 64-QAM.

Although the EVM calculation is not related to different modulations and gives the same result for different modulations, the SER calculation gives quite different results for different modulations. Because 64-QAM has much more points in constellation than QPSK, which means that the decision zones of 64-QAM are much smaller than those of QPSK, intuitively, if the same sample cloud is received for a certain 


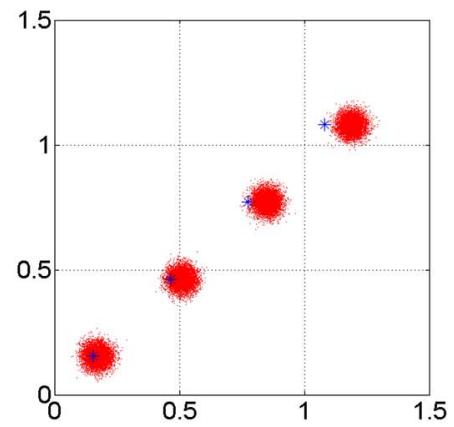

Transmitter amplitude imbalance with $k=1.1=0.83 \mathrm{~dB}, E_{\mathrm{s}} / N_{0}=25 \mathrm{~dB}$

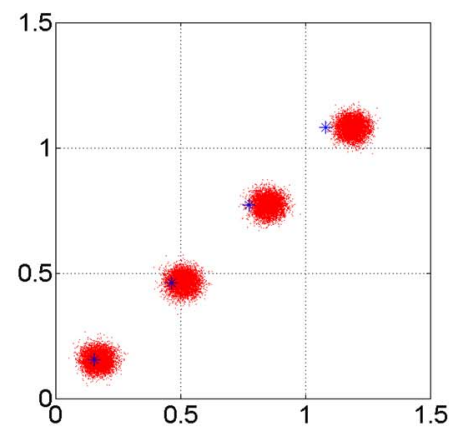

Receiver amplitude imbalance with $l=1.1=0.83 \mathrm{~dB}, E_{\mathrm{s}} / N_{0}=25 \mathrm{~dB}$

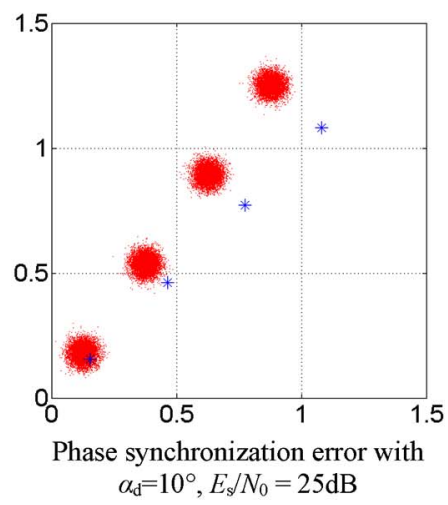

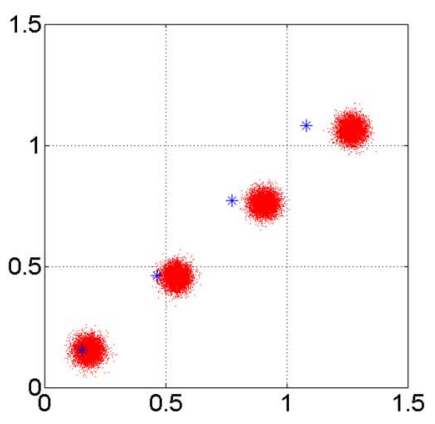

Transmitter phase imbalance with $\varphi=10^{\circ}, E_{\mathrm{s}} / N_{0}=25 \mathrm{~dB}$
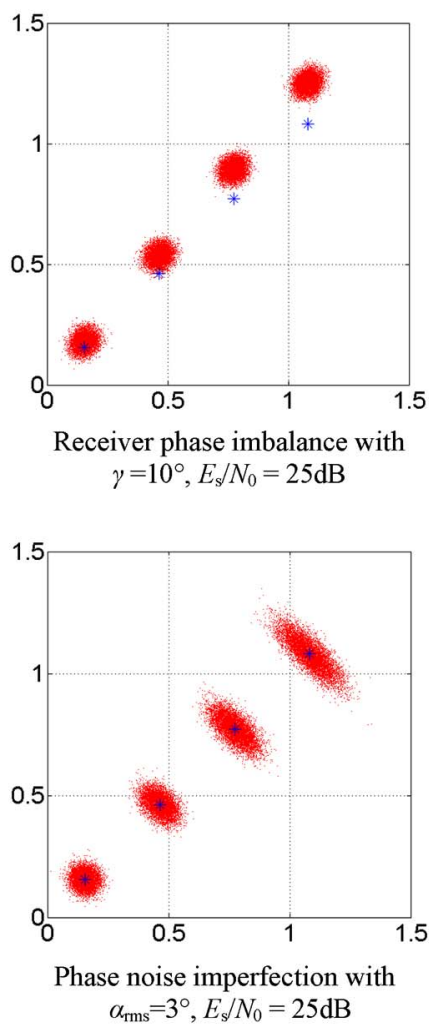

- Rx samples * Tx symbols

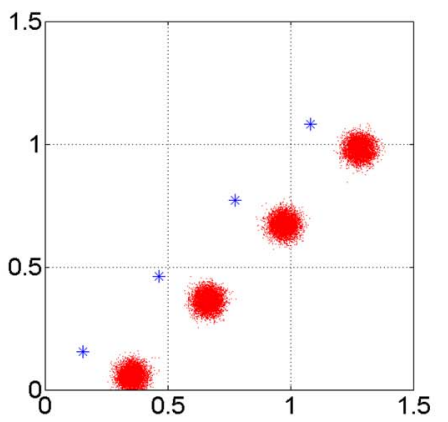

Transmitter offset imperfection with $\mathbf{a}=[0.2,-0.1]^{\mathrm{T}}, E_{\mathrm{s}} / N_{0}=25 \mathrm{~dB}$

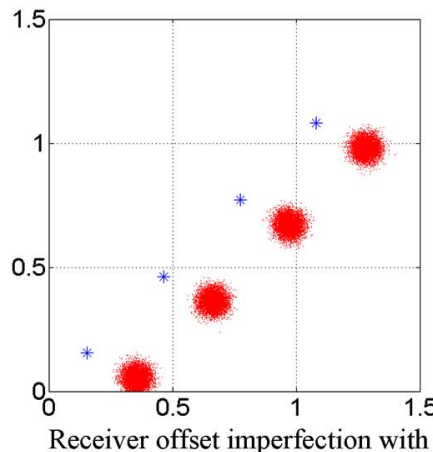

Receiver offset imperfection with $\mathbf{b}=[0.2,-0.1]^{\mathrm{T}}, E_{\mathrm{s}} / N_{0}=25 \mathrm{~dB}$

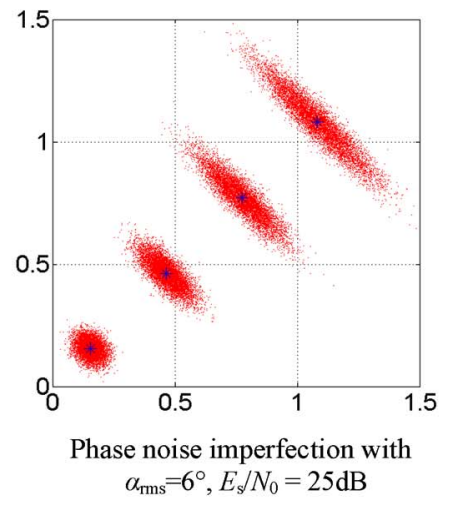

Fig. 6. 64-QAM constellations affected by different imperfections.

point, more samples drop out of the decision zone and yield more error symbols for 64-QAM. Therefore, the SER of 64-QAM is more sensitive to each imperfection.

Fig. 10 shows the SER variation with $E_{s} / N_{0}$ in which only the transmitter phase imbalance is considered. For an SER of $10^{-4}$, the $3^{\circ}$ phase imbalance yields about 3-dB degradation for 64-QAM and about 1-dB degradation for 16-QAM but yields ignorable degradation for QPSK. The figure also shows that if the phase imbalance is increased to certain value such as $44^{\circ}$ for QPSK, $15^{\circ}$ for $16-\mathrm{QAM}$, and $7^{\circ}$ for $64-\mathrm{QAM}$, the SER curve becomes almost flat in the low- $E_{s} / N_{0}$ area. Those angles agree with half of the smallest angle difference between the points with the same amplitude in each modulation scheme, i.e., $45^{\circ}$ for QPSK, $18.4^{\circ}$ for $16-\mathrm{QAM}$, and $8.1^{\circ}$ for 64-QAM. Several data points obtained from simulation are shown to verify the theoretical results.
Fig. 11 shows the SER variation with $E_{s} / N_{0}$ in which only the deterministic phase synchronization error $\alpha_{d}$ is considered. This figure gives curves similar to those in Fig. 10. However, the angle in the phase synchronization error degrades the SER a little bit more than the same angle in the transmitter phase imbalance.

Fig. 12 considers only the phase noise contribution. It shows that the phase noise degrades the SER more than the phase imbalance and the deterministic phase synchronization error. The curves also show the noise floors that are related not only to the variance $\alpha_{\mathrm{rms}}$ but also to the different modulation schemes. For 64-QAM, a phase noise with $3^{\circ}$ variance will yield a noise floor above $10^{-3}$, which is unacceptable for most systems. Therefore, the phase noise is the primary design consideration among the parameters discussed in this paper for a system using 64-QAM. The last curve list in the legend is for 64-QAM 


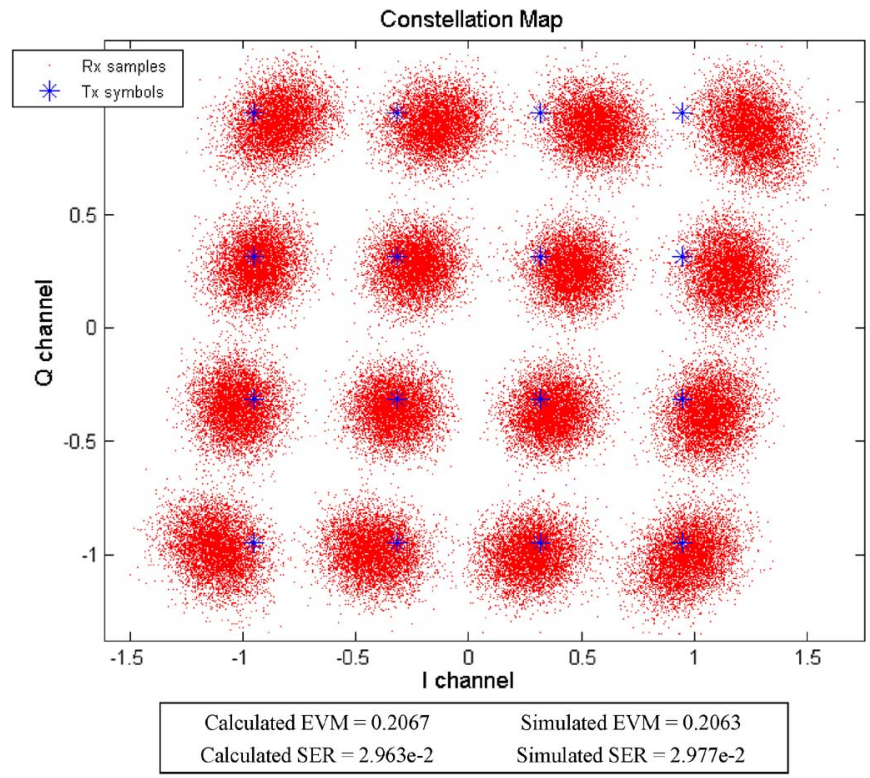

Fig. 7. 16-QAM sample situation: $k=1.05=0.42 \mathrm{~dB}, \varphi=5^{\circ}, \mathbf{a}=$ $[0.06,0]^{T}, l=1.05=0.42 \mathrm{~dB}, \gamma=2^{\circ}, \mathbf{b}=[0,-0.05]^{T}, \alpha_{d}=-3^{\circ}$, $\alpha_{\mathrm{rms}}=3^{\circ}$, and $N_{0}=-15 \mathrm{~dB}$ with $1 \mathrm{e} 5$ symbols simulated.

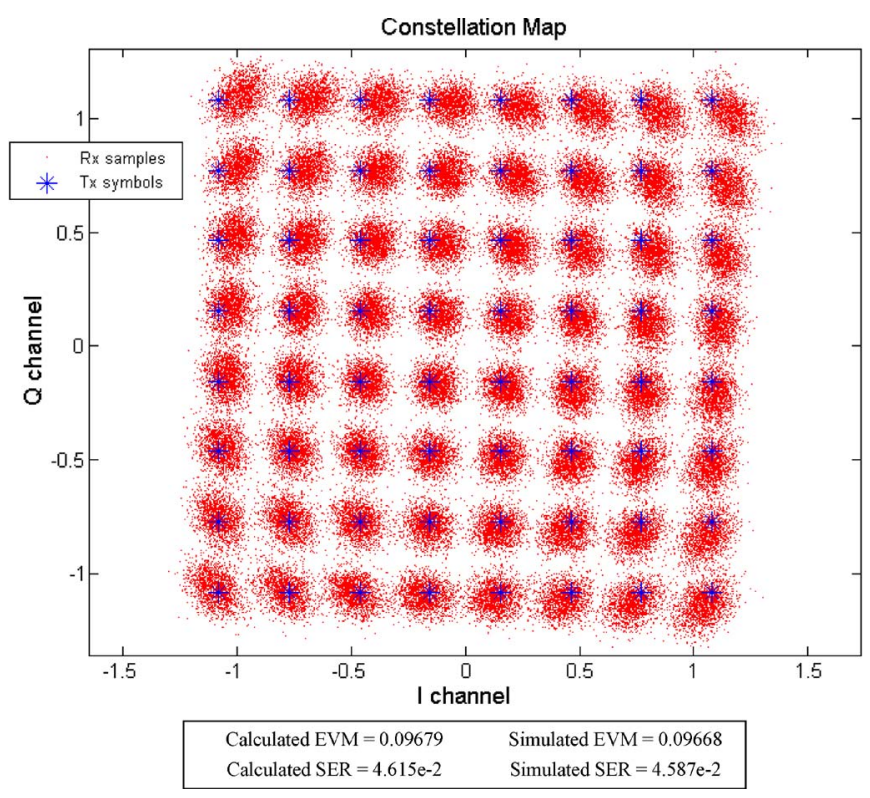

Fig. 8. 64-QAM sample situation: $k=1.02=0.17 \mathrm{~dB}, \varphi=2^{\circ}, \mathbf{a}=$ $[0.03,0]^{T}, l=0.97=-0.26 \mathrm{~dB}, \gamma=-1^{\circ}, \mathbf{b}=[0,-0.02]^{T}, \alpha_{d}=-1^{\circ}$, $\alpha_{\mathrm{rms}}=2^{\circ}$, and $N_{0}=-20 \mathrm{~dB}$ with 1e5 symbols simulated.

with $\alpha_{\mathrm{rms}}=0.5^{\circ}$, which is a typical phase noise come from a WLAN synthesizer [8]. The result shows that this phase noise degrades the system performance by less than $1 \mathrm{~dB}$.

If more than one parameter is considered, the results will be much more complicated and can hardly be shown in one plot. For designing a real system, usually, a specific system performance is required, and under this system requirement, the contribution of each component in the system is investigated, and the link budget is calculated and distributed to each component. In Fig. 13, a simple example is shown for a system using 64-QAM and requiring an SER performance of $10^{-4}$, which is a typical requirement for a wireless communication system. The 3-D surface shows the minimum $E_{s} / N_{0}$ to achieve
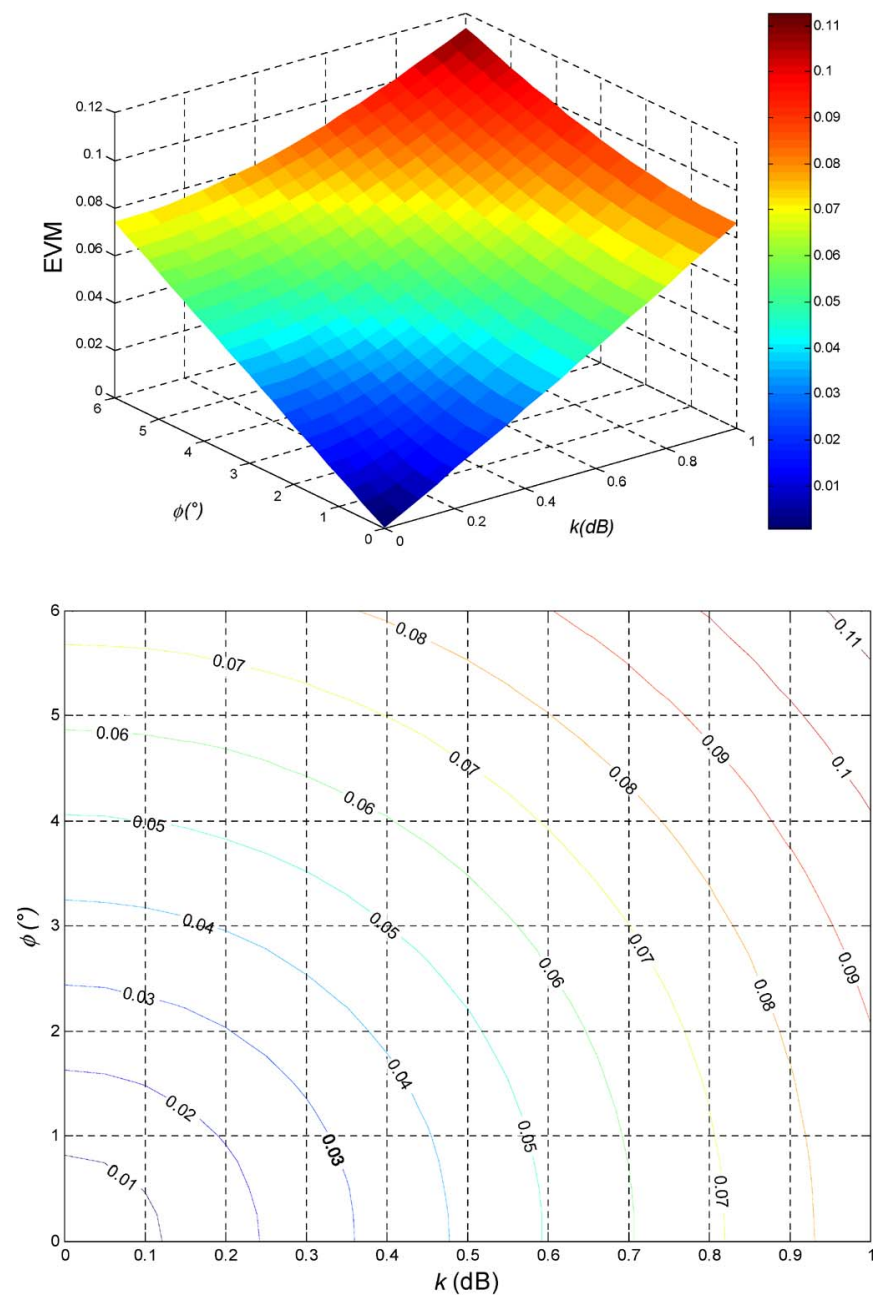

Fig. 9. EVM variation with the transmitter amplitude and phase imbalance for $M$-QAM. Top: 3-D diagram, bottom: 2-D contour.

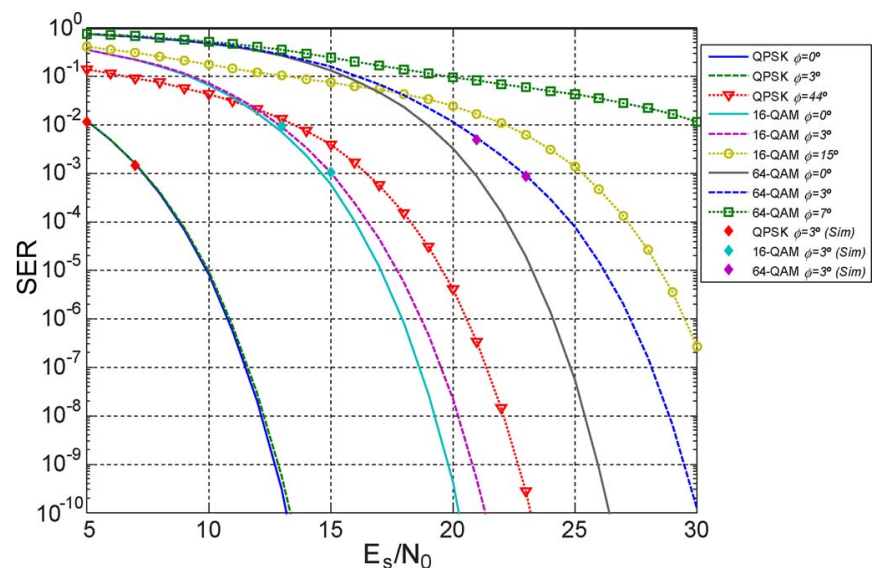

Fig. 10. SER $\sim E_{\mathrm{S}} / N_{0}$ with different transmitter phase imbalance for QPSK, 16-QAM, and 64-QAM.

the SER requirement in the presence of phase noise and phase synchronization error.

In another case, for a WLAN system using 64-QAM, assuming that the channel noise can be neglected, that synchronization is ideal, and that the transmitter and the receiver have the same imbalances, the proposed theoretical model can be used to find the maximum tolerable phase and amplitude 


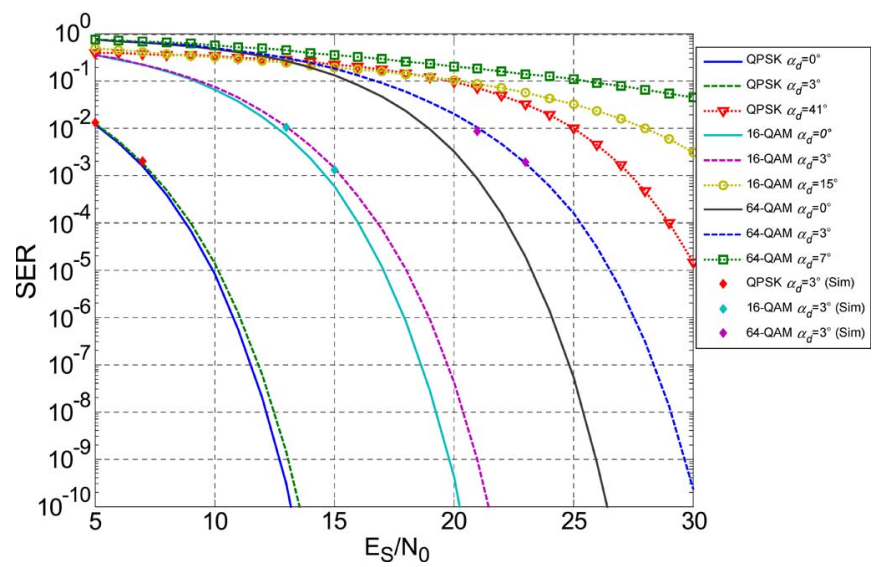

Fig. 11. SER $\sim E_{\mathrm{s}} / N_{0}$ with different phase synchronization errors for QPSK, 16-QAM, and 64-QAM.

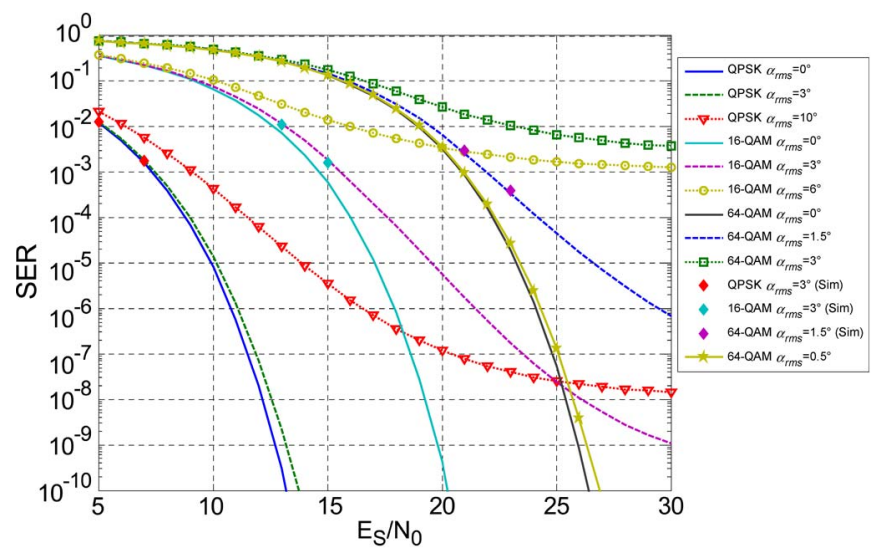

Fig. 12. SER $\sim E_{\mathrm{S}} / N_{0}$ with phase noise for QPSK, 16-QAM, and 64-QAM.

imbalance and LO phase noise for a typical system requirement of SER $=10^{-4}$, as shown in Fig. 14. It should be pointed out that the Q-factor of on-chip resonators can greatly be improved with the advance of integrated microelectromechanical system technology [15], which will benefit the integrated RF filter and oscillator performance for wireless transceiver designs. Nevertheless, the methodology presented in this paper provides an efficient means and guideline for system and circuit designers to find a proper tradeoff between the imbalances and phase noise requirements for various wireless applications such as WiFi [13] and wireless sensor networks [16]-[18].

\section{CONCLUSION}

In the paper, a theoretical analysis of the joint effects of the transmitter and receiver phase and amplitude imbalance, phase noise, and channel noise on $M$-QAM systems has been presented. An analytic expression for the EVM and an integral expression for the SER have been derived in (36) and (40), which provides an efficient means to specify the wireless system and block requirements considering the effects of those imperfections on the system performance. The analysis showed that 64-QAM is very sensitive to phase noise and other system imperfections. Therefore, the link budget should carefully be considered and distributed to each component for a 64-QAM system. The analytical results were also compared with time-
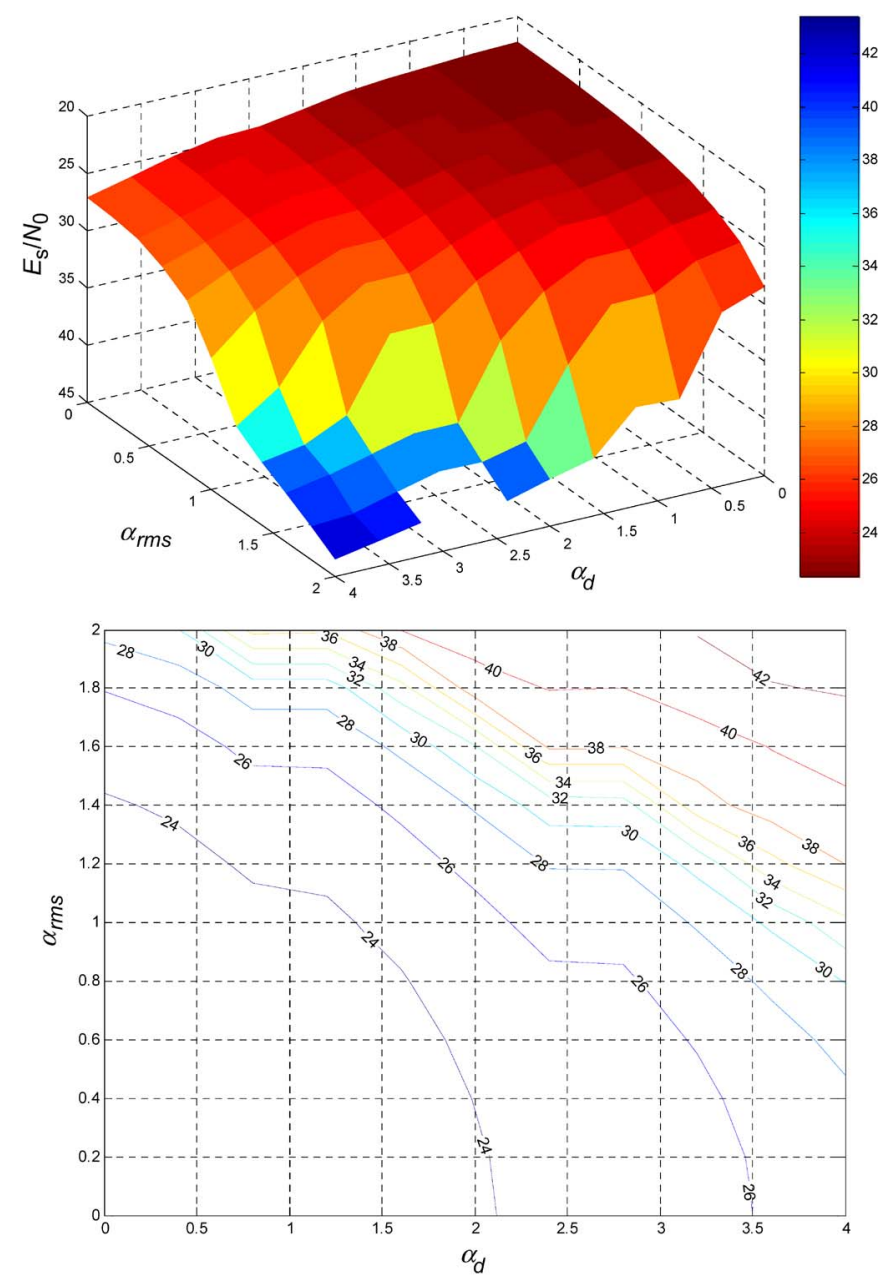

Fig. 13. $E_{\mathrm{S}} / N_{0}$ requirement for $10^{-4}$ SER with phase noise and phase synchronization error. Top: 3-D diagram, bottom: 2-D contour.

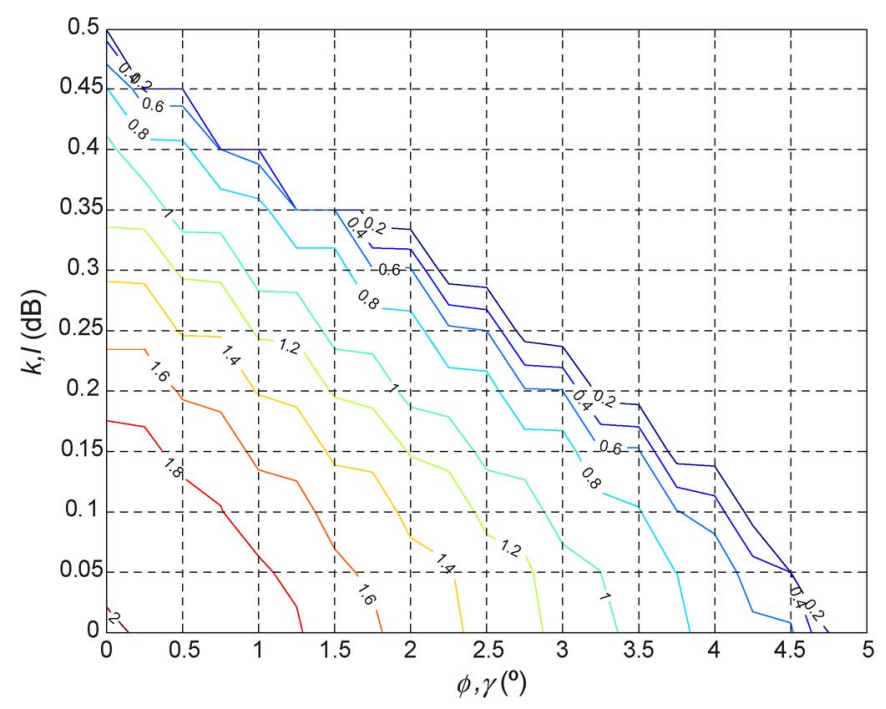

Fig. 14. Tolerable phase and amplitude imbalance and LO phase noise for SER $=10^{-4}$ in a 64-QAM WLAN system.

consuming Monte Carlo simulation results and showed a good agreement. For a more complete system analysis, the nonlinearity from the RF amplifier and the multipath fading channel should be considered. 


\section{APPENDIX}

\section{Channel Noise Covariance Matrix}

Assuming an ideal LPF that has a pass bandwidth of $B$ and the impulse response of $h(t)$, the received noise in two channels can then be expressed as

$$
\begin{aligned}
n_{i}(t) & =l[n(t) \cos (\omega t+\alpha)] \otimes h(t) \\
& =l \int_{\tau=0}^{+\infty} n(t-\tau) \cos (\omega t-\omega \tau+\alpha) h(\tau) d \tau \\
n_{q}(t) & =[n(t) \sin (\omega t+\alpha+\gamma)] \otimes h(t) \\
& =\int_{\tau=0}^{+\infty} n(t-\tau) \sin (\omega t-\omega \tau+\alpha+\gamma) h(\tau) d \tau
\end{aligned}
$$

where $\mathbf{n}_{r}(t) \triangleq\left[n_{i}(t), n_{q}(t)\right]^{\mathrm{T}}, \otimes$ denotes convolution, $n(t)$ is the white Gaussian noise in the channel that has a double-side PSD of $N_{0} / 2$. Then, each element in the covariance matrix is obtained as follows:

$$
\begin{aligned}
E\left[n_{i}^{2}(t)\right]= & l^{2} E\left[\int_{\tau=-\infty}^{+\infty} n(t-\tau) \cos (\omega t-\omega \tau+\alpha) h(\tau) d \tau \int_{\xi=-\infty}^{+\infty}\right. \\
= & l^{2} \int_{\tau=-\infty}^{+\infty} \int_{\xi=-\infty}^{+\infty} E[n(t-\xi) \cos (\omega t-\omega \xi+\alpha) h(\xi) d \xi] \\
& \times \cos (\omega t-\omega \tau+\alpha) h(\xi) h(\tau) d \xi d \tau \\
= & l^{2} \int_{\tau=-\infty}^{+\infty} \int_{\xi=-\infty}^{+\infty} \frac{N_{0}}{2} \delta(\xi-\tau) \cos (\omega t-\omega \xi+\alpha) \\
& \times \cos (\omega t-\omega \tau+\alpha) h(\xi) h(\tau) d \xi d \tau \\
= & l^{2} \frac{N_{0}}{2} \int_{\tau=-\infty}^{+\infty}\left[\frac{1}{2}+\frac{1}{2} \cos (2 \omega t-2 \omega \tau+\alpha)\right. \\
= & l^{2} \frac{N_{0}}{4} \int_{\tau=-\infty}^{+\infty} h^{2}(\tau) d \tau+A_{r}^{2} l^{2} \frac{N_{0}}{4} \int_{\tau=-\infty}^{+\infty} \\
& \times \cos (2 \omega t-2 \omega \tau+2 \alpha) h^{2}(\tau) d \tau \\
= & l^{2} N_{0} B / 4
\end{aligned}
$$

From the Plancherel's theorem

$$
\begin{aligned}
\int_{\tau=-\infty}^{+\infty} h^{2}(\tau) d \tau & =\int_{f=-\infty}^{+\infty}|H(f)|^{2} d f=B \\
H(f) & = \begin{cases}1, & |f|<B / 2 \\
0, & \text { otherwise }\end{cases}
\end{aligned}
$$

where $H(f)$ is the frequency response of the ideal LPF. Furthermore, assuming that the carrier frequency is much larger than the passband of the LPF $\omega \gg \pi B$, the integral $\int_{\tau=-\infty}^{+\infty} \cos (2 \omega t-2 \omega \tau+2 \alpha) h^{2}(\tau) d \tau$ is approximately equal to zero.
Similarly,

$$
\begin{aligned}
E & {\left[n_{q}^{2}(t)\right] } \\
& =E\left[\int_{\tau=0}^{+\infty} n(t-\tau) \sin (\omega t-\omega \tau+\alpha+\gamma) h(\tau) d \tau\right. \\
& \left.\int_{\xi=0}^{+\infty} n(t-\xi) \sin (\omega t-\omega \xi+\alpha+\gamma) h(\xi) d \xi\right] \\
& =N_{0} B / 4 . \\
& =l E\left[\int_{\tau=0}(t) n_{q}(t)\right] \\
& =\frac{l N_{0}}{2} \int_{\tau=0}^{+\infty}\left[\frac{1}{2} \sin (2 \omega t-2 \omega \tau+\cos (\omega t-\omega \tau+\alpha) h(\tau) d \tau\right. \\
& =\frac{l N_{0} B}{4} \sin (\gamma) .
\end{aligned}
$$

From (47), (49), and (50), the covariance matrix is expressed as

$$
\mathbf{C}_{\mathbf{n}_{r} \mathbf{n}_{r}}=\frac{N_{0} B}{4}\left[\begin{array}{cc}
l^{2} & l \sin (\gamma) \\
l \sin (\gamma) & 1
\end{array}\right] .
$$

\section{REFERENCES}

[1] D. B. Leeson, "A simple model of feedback oscillator noise spectrum," Proc. IEEE, vol. 54, no. 2, pp. 329-330, Feb. 1966.

[2] T. H. Lee and A. Hajimiri, "Oscillator phase noise: A tutorial," IEEE J. Solid-State Circuits, vol. 35, no. 3, pp. 326-336, Mar. 2000.

[3] T. S. Rappaport, Wireless Communications Principles \& Practice. Englewood Cliffs, NJ: Prentice-Hall, 1996.

[4] A. Hajimiri and T. H. Lee, "A general theory of phase noise in electrical oscillators," IEEE J. Solid-State Circuits, vol. 33, no. 2, pp. 179-194, Feb. 1998.

[5] B. Razavi, "A study of phase noise in CMOS oscillators," IEEE. J. SolidState Circuits, vol. 31, no. 3, pp. 331-343, Mar. 1996.

[6] A. Hajimiri, S. Limotyrakis, and T. H. Lee, "Jitter and phase noise in ring oscillators," IEEE J. Solid-State Circuits, vol. 34, no. 6, pp. 790-804, Jun. 1999.

[7] K. A. Kouznetsov and R. G. Meyer, "Phase noise in LC oscillators," IEEE J. Solid-State Circuits, vol. 35, no. 8, pp. 1244-1248, Aug. 2000.

[8] W. M. Rogers, F. F. Dai, C. Plett, and M. S. Cavin, "Design and characterization of a $5.2 \mathrm{GHz} / 2.4 \mathrm{GHz} \Sigma \Delta$ fractional-N frequency synthesizer for low-phase noise performance," EURASIP J. Wireless Commun. Netw., vol. 2006, pp. 1-11, 2006, Article ID 48489.

[9] J. K. Cavers and M. W. Liao, "Adaptive compensation for imbalance and offset losses in direct conversion transceivers," IEEE Trans. Veh. Technol. vol. 42, no. 4, pp. 581-588, Nov. 1993.

[10] E. Costa and S. Pupolin, "M-QAM-OFDM system performance in the presence of nonlinear amplifier and phase noise," IEEE Trans. Commun., vol. 50, no. 3, pp. 462-472, Mar. 2002.

[11] A. Georgiadis, "Gain, phase imbalance, and phase noise effects on error vector magnitude," IEEE Trans. Veh. Technol., vol. 53, no. 2, pp. 443-449, Mar. 2004.

[12] A. Netsell, "Interpret and apply EVM to RF system design," Microw. RF, vol. 40, no. 12, pp. 83-94, Dec. 2001.

[13] D. G. Rahn, M. S. Cavin, F. F. Dai, N. Fong, R. Griffith, J. Macedo, D. Moore, J. W. M. Rogers, and M. Toner, "A fully integrated multi-band MIMO WLAN transceiver RFIC," IEEE J. Solid-State Circuits, vol. 40, no. 8, pp. 1629-1641, Aug. 2005. 
[14] T. Emura and L. Wang, "A high-resolution interpolator for incremental encoders based on the quadrature PLL method," IEEE Trans. Ind. Electron., vol. 47, no. 1, pp. 84-90, Feb. 2000.

[15] M. M. Shalaby, M. A. Abdelmoneum, and K. Saitou, "Design of spring coupling for high-Q high-frequency MEMS filters for wireless applications," IEEE Trans. Ind. Electron., vol. 56, no. 4, pp. 1022-1030, Apr. 2009.

[16] V. C. Gungor and G. P. Hancke, "Industrial wireless sensor networks: Challenges, design principles, and technical approaches," IEEE Trans. Ind. Electron., vol. 56, no. 10, pp. 4258-4265, Oct. 2009.

[17] B. Lu and V. C. Gungor, "Online and remote motor energy monitoring and fault diagnostics using wireless sensor networks," IEEE Trans. Ind. Electron., vol. 56, no. 10, pp. 4651-4659, Oct. 2009.

[18] K. Al Agha, M.-H. Bertin, T. Dang, A. Guitton, P. Minet, T. Val, and J.-B. Viollet, "Which wireless technology for industrial wireless sensor networks? The development of OCARI technology," IEEE Trans. Ind. Electron., vol. 56, no. 10, pp. 4266-4278, Oct. 2009.

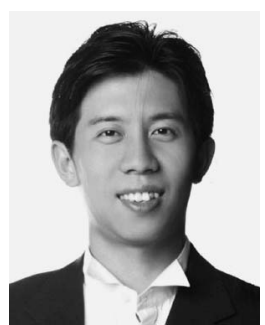

ZhenQi Chen (S'02) was born in Beijing, China, in 1978. He received the B.S. and M.S. degrees in electronic engineering from Tsinghua University, Beijing, China, in 2000 and 2003, respectively. $\mathrm{He}$ is currently working toward the Ph.D. degree in electrical engineering at Auburn University, Auburn, AL.

He is also currently a Design Engineer with Qualcomm Inc. His research interests include digital and mixed-signal circuit design and wireless communications.

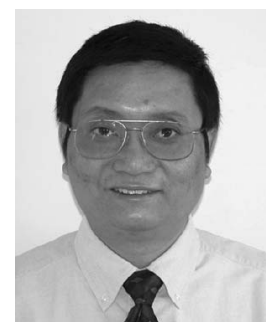

Fa Foster Dai (M'92-SM'00-F'09) received the $\mathrm{Ph} . \mathrm{D}$. degree in electrical and computer engineering from Auburn University, Auburn, AL, in 1997, and the $\mathrm{Ph} . \mathrm{D}$. degree in electrical engineering from Pennsylvania State University, University Park, in 1998.

From 1997 to 2000, he was with Hughes Network Systems of Hughes Electronics, Germantown, MD, where he was a Member of Technical Staff in very large scale integration (VLSI), designing analog and digital ICs for wireless and satellite communications. From 2000 to 2001, he was with YAFO Networks, Hanover, MD, where he was a Technical Manager and a Principal Engineer in VLSI designs, leading high-speed SiGe IC designs for fiber communications. From 2001 to 2002, he was with Cognio Inc., Gaithersburg, MD, designing radio frequency (RF) ICs for integrated multiband MIMO wireless transceivers. From 2002 to 2004, he was an RFIC consultant for Cognio Inc. In August 2002, he joined Auburn University, where he is currently a Professor of electrical and computer engineering. He is a coauthor of the book Integrated Circuit Design for High-Speed Frequency Synthesis (Artech House, 2006). He is the holder of five U.S. patents. His research interests include VLSI circuits for analog and mixed-signal applications, RFIC designs for wireless and broadband networks, ultrahigh-frequency synthesis, and mixed-signal built-in self-test (BIST).

Dr. Dai received the Research Award for Excellence from the College of Engineering of Auburn University in 2009. He has served as a Guest Editor for the IEEE TRANSACtions ON INdUStrial Electronics in 2001, 2009, and 2010. He served on the Technical Program Committees of the IEEE Symposium on VLSI Circuits from 2005 to 2008. He currently serves on the Technical Program Committees of the IEEE Custom Integrated Circuits Conference (CICC) and the IEEE Bipolar/BiCMOS Circuits and Technology Meeting (BCTM). 